\title{
circPTPN4 regulates myogenesis via the miR-499-3p/NAMPT axis
}

Bolin Cai ${ }^{1,2}$, Manting Ma ${ }^{1,2}$, Zhen Zhou ${ }^{1,2}$, Shaofen Kong ${ }^{1,2}$, Jing Zhang ${ }^{1,2}$, Xiquan Zhang ${ }^{1,2}$ and Qinghua Nie $e^{1,2^{*}}$ (D)

\begin{abstract}
Background: Circular RNAs (circRNAs) are a novel class of endogenous ncRNA, which widely exist in the transcriptomes of different species and tissues. Recent studies indicate important roles for circRNAs in the regulation of gene expression by acting as competing endogenous RNAs (ceRNAs). However, the specific role of circRNAs in myogenesis is still poorly understood. In this study, we attempted to systematically identify the circRNAs involved in myogenesis and analyze the biological functions of circRNAs in chicken skeletal muscle development.
\end{abstract}

Results: In total, 532 circRNAs were identified as being differentially expressed between pectoralis major (PEM) and soleus (SOL) in 7-week-old Xinghua chicken. Among them, a novel circRNA (novel_circ_002621), generated by PTPN4 gene, was named circPTPN4 and identified. circPTPN4 is highly expressed in skeletal muscle, and its expression level is upregulated during myoblast differentiation. circPTPN4 facilitates the proliferation and differentiation of myoblast. Moreover, circPTPN4 suppresses mitochondria biogenesis and activates fast-twitch muscle phenotype. Mechanistically, circPTPN4 can function as a ceRNA to regulate NAMPT expression by sponging miR-499-3p, thus participating in AMPK signaling.

Conclusions: circPTPN4 functions as a ceRNA to regulate NAMPT expression by sponging miR-499-3p, thus promoting the proliferation and differentiation of myoblast, as well as activating fast-twitch muscle phenotype.

Keywords: Chicken, CircPTPN4, Circular RNA, MiR-499-3p, Myogenesis, NAMPT, The transformation of myofiber

\section{Background}

Chicken is the second most consumed meat in China, and the meat production performance of chicken determines its commercial value. While increasing the yield, improving the quality of chicken is the direction that poultry breeders have been working hard on. Recently, it has come to light that the composition of myofiber types has an important relationship with muscle quality $[1,2]$. The discovery of genetic regulatory factors involved in

\footnotetext{
* Correspondence: nqinghua@scau.edu.cn

'State Key Laboratory for Conservation and Utilization of Subtropical Agro-Bioresources, Lingnan Guangdong Laboratory of Agriculture, College of Animal Science, South China Agricultural University, Guangzhou 510642, Guangdong, China

${ }^{2}$ Guangdong Provincial Key Lab of Agro-Animal Genomics and Molecular Breeding, and Key Laboratory of Chicken Genetics, Breeding and

Reproduction, Ministry of Agriculture, Guangzhou 510642, Guangdong, China
}

skeletal muscle development is of great significance to chicken production.

Gene is the carrier of genetic information, carrying various biological processes of life. The product, such as peptide or protein molecules, plays a key role in it [3]. However, protein-encoding genes only account for a small portion $(\sim 2 \%)$ of the genome, while more than $98 \%$ of the genomic loci are transcribed to noncoding RNAs (ncRNAs) [4]. Skeletal muscle is the largest tissue in the body, which comprises about $40 \%$ of the total body mass. The development of skeletal muscle is closely related to growth and health, and is directly regulated by multiple genetic factors. Noticeably, recent studies have found that ncRNAs play critical roles in it $[5,6]$.

(c) The Author(s). 2022 Open Access This article is licensed under a Creative Commons Attribution 4.0 International License, which permits use, sharing, adaptation, distribution and reproduction in any medium or format, as long as you give appropriate credit to the original author(s) and the source, provide a link to the Creative Commons licence, and indicate if changes were made. The images or other third party material in this article are included in the article's Creative Commons licence, unless indicated otherwise in a credit line to the material. If material is not included in the article's Creative Commons licence and your intended use is not permitted by statutory regulation or exceeds the permitted use, you will need to obtain permission directly from the copyright holder. To view a copy of this licence, visit http://creativecommons.org/licenses/by/4.0/ The Creative Commons Public Domain Dedication waiver (http://creativecommons.org/publicdomain/zero/1.0/) applies to the data made available in this article, unless otherwise stated in a credit line to the data. 
Circular RNAs (circRNAs) are a novel class of endogenous ncRNA with a covalently closed loop, which widely exist in the transcriptomes of different species and tissues [7, 8]. Compared with linear RNA (such as long noncoding RNA), circRNA has higher structural stability and conservation. It is becoming increasingly clear that circRNAs can widely be involved in a series of biological processes by acting as a miRNA sponge, participating in regulating the expression of its own linear RNA in different ways, coding protein, and deriving pseudogenes [9-12]. Although, more and more circRNAs have been found by high-throughput sequencing, the mechanism of circRNA regulation involved in skeletal muscle development is still poorly understood.

MicroRNAs (miRNAs) are endogenous noncoding single-stranded RNA molecules of 18-22 nt long that are capable of degrading or inhibiting target mRNAs by perfect or imperfect pairing with the 3' untranslated region ( $3^{\prime}$ UTR) of the target mRNA to regulate posttranscriptional gene expression [13, 14]. Recent study has found that miR-499-3p could suppress retinal cell proliferation while promote apoptosis to induce diabetic retinopathy by enhancing activation of the TLR4 signaling pathway [15]. In pigs, the expression of ssc-miR-499$3 p$ was significantly correlated to the expression of myoglobin and $\mathrm{pH}$, prompting its potential regulatory role in skeletal muscle fiber transformation and meat quality traits [16]. However, the exact biological function of miR-499-3p in skeletal muscle development has not been reported yet.

Nicotinamide phosphoribosyltransferase (NAMPT) is the rate-limiting enzyme which catalyzes the conversion of nicotinamide and phosphoribosyl-pyrophosphates to nicotinamide mononucleotide in the mammalian nicotinamide adenine dinucleotide $\left(\mathrm{NAD}^{+}\right)$synthetic salvage pathway $[17,18]$. Recently, numerous studies have indicated that NAMPT is able to modulate processes involved in the pathogenesis of obesity and related disorders by influencing the oxidative stress response, apoptosis, lipid and glucose metabolism, inflammation and insulin resistance [19]. But little is known about how NAMPT functions in skeletal muscle development.

In this study, to systematically identify the circRNAs involved in skeletal muscle development, pectoralis major (PEM) and soleus (SOL) in 7-week-old Xinghua chicken were used for circRNA sequencing (circRNAseq). Based on this result, a novel circRNA (novel_circ 002621), generated by the PTPN4 gene, was identified and named $\operatorname{circPTPN4}$. circPTPN4 is highly expressed in skeletal muscle, and its expression upregulates with myoblast differentiation. Functional studies demonstrated that $\operatorname{circPTPN4}$ promotes the proliferation and differentiation of myoblast, as well as activates the fasttwitch muscle phenotype. Furthermore, the mechanistic investigation revealed that circPTPN4 can function as a competing endogenous RNA (ceRNA) by sponging $m i R$ $499-3 p$, thus regulating the expression of NAMPT to mediate the AMPK signaling.

\section{Methods}

\section{Ethics statement}

All animal experimental protocols were conformed to "The Instructive Notions with Respect to Caring for Laboratory Animals" issued by the Ministry of Science and Technology of the People's Republic of China, and approved by the Institutional Animal Care and Use Committee at the South China Agricultural University (approval ID: 2021-C018).

\section{Animals and cells}

Seven-week-old Xinghua female chickens were hatched from the Avian Farm of South China Agricultural University (Guangzhou, China). The chickens were euthanized, and organs and tissues were collected after rapid dissection, then immediately frozen in liquid nitrogen and stored at $-80^{\circ} \mathrm{C}$.

Chicken primary myoblasts (CPMs) were isolated from leg muscles of E11 (11-embryonic-day-old) chicken and cultured as previously described [20]. Firstly, the muscle tissues were dissected away from the skin and bone, and then homogenized in a petri dish. To release single cells, the suspension was digested with pancreatin for $20 \mathrm{~min}$ at $37^{\circ} \mathrm{C}$. After neutralization with complete medium, single cells were collected by centrifugation at $500 \times g$. Subsequently, serial plating was performed to enrich primary myoblasts and eliminate fibroblasts. Primary myoblasts were cultured in Roswell Park Memorial Institute (RPMI)-1640 medium (Gibco, MD, USA) with 20\% FBS, $1 \%$ nonessential amino acids, and $0.2 \%$ penicillin/ streptomycin. The purity of isolated primary myoblasts was verified by immunofluorescence (Fig. S1).

To induce myogenic differentiation, the growth medium was removed and replaced with differentiation medium (RPMI-1640 medium [Gibco, MD, USA] containing $2 \%$ horse serum) after myoblasts achieved $90 \%$ cell confluence.

\section{Circular RNA sequencing (circRNA-seq)}

The pectoralis major (PEM; which is mainly composed of fast-twitch fibers) and soleus (SOL; which has higher proportion of slow muscle fibers) of 7-week-old Xinghua chicken were used for circRNA-seq. After extraction, total RNAs were treated with RNase $\mathrm{R}$ to degrade the linear RNAs, and purified using RNeasy MinElute Cleanup Kit (Qiagen, Walldorf, Germany). Next, strandspecific library was constructed using VAHTS Total RNA-seq (H/M/R) Library Prep Kit for Illumina following the manufacturer's instructions. Briefly, ribosome 
RNAs were removed to retain circRNAs. The enriched circRNAs were fragmented into short fragments by using fragmentation buffer and reverse transcripted into cDNA with random primers. Second-strand cDNA were synthesized by DNA polymerase I, RNase $\mathrm{H}$, dNTP (dUTP instead of dTTP) and buffer. Next, the cDNA fragments were purified with VAHTSTM DNA Clean Beads, end repaired, poly(A) added, and ligated to Illumina sequencing adapters. Then UNG (Uracil-N-Glycosylase) was used to digest the second-strand cDNA. The digested products were purified with VAHTSTM DNA Clean Beads, PCR amplified, and sequenced using Illumina HiSeq 2500 by Gene Denovo Biotechnology Co. (Guangzhou, China). The raw data of circRNA-seq were deposited in the Sequence Read Archive (SRA) database under accession no. PRJNA751251.

Parental genes of differentially expressed circRNAs were subjected to enrichment analysis of Gene Ontology (GO) functions and Kyoto Encyclopedia of Genes and Genomes (KEGG) pathways.

\section{Validation of circRNA}

The circRNAs were validated using PCR with divergent and convergent primers as previously described [21]. To confirm the junction sequence of circRNAs, PCR products of divergent primers were gel purified and submitted for Sanger sequencing at Tsingke Biotechnology Co., Ltd. (Beijing, China). To check the sensitivity of circRNA to RNase R, quantitative PCR was also performed using RNA samples with and without RNase $\mathrm{R}$ treatment. Primers used for the validation of circRNA are summarized in Table S1.

\section{RNA extraction, CDNA synthesis, and quantitative real- time PCR}

Total RNA was extracted by using the TRIzol reagent (TaKaRa, Otsu, Japan), following the manufacturer's protocol. Nuclear and cytoplasmic RNA fractionation was performed by using the Paris kit (Ambion, Life Technologies, Carlsbad, CA, USA) as recommended by the supplier. The PrimeScript RT Reagent Kit with gDNA Eraser (Perfect Real Time) (TaKaRa, Otsu, Japan) was used to synthesize cDNA. Quantitative real-time PCR was performed as described before [22]. And primers used for quantitative real-time PCR are listed in Table S1.

\section{Plasmid construction and RNA oligonucleotides}

For pGL3 luciferase reporter vectors construction, the active region of PTPN4 gene promoter containing FOXA2 binding site and FOXA2 binding site mutant were amplified and cloned into the pGL3-Basic Vector (Promega, Madison, WI, USA) by using XhoI and HindIII restriction sites.
For FOXA2 expression vectors construction, the coding sequence of FOXA2 was amplified by PCR, and then subcloned into HindIII and XhoI restriction sites of the pcDNA3.1-3xFLAG-C vector or cloned into the expression plasmid pcDNA-3.1 (Promega, Madison, WI, USA) by using HindIII and XhoI restriction sites.

For circPTPN4 overexpression vector construction, the linear sequence of $\operatorname{circPTPN} 4$ was amplified and then subcloned into EcoRI and BamHI restriction sites of the pCD25-ciR vector (Geneseed Biotech, Guangzhou, China) by using the Trelief ${ }^{\mathrm{m}}$ SoSoo Cloning Kit (Tsingke Biotech, Beijing, China), following the manufacturer's protocol.

For pmirGLO dual-luciferase miRNA target reporter vector, the segment sequences of $\operatorname{circPTPN4}$ and NAMP $T$ 3' untranslated region (UTR) that contained the putative $m i R-499-3 p$ binding sequence were amplified by PCR, and then subcloned into XhoI and SalI restriction sites in the pmirGLO dual luciferase reporter vector (Promega, Madison, WI, USA). Mutant plasmids were generated by changing the binding site of $m i R-499-3 p$ from GTGATGT to TGTCGTG.

miR-499-3p mimic, mimic negative control (NC), 3' end biotinylated miR-499-3p mimic, $3^{\prime}$ end biotinylated mimic NC and small interfering RNA (siRNA) against circPTPN4 were designed and synthesized by Guangzhou RiboBio (Guangzhou, China).

The primers and oligonucleotide sequences used in this study are listed in Tables S1 and S2.

\section{Cell transfection}

All transient transfections were performed with Lipofectamine 3000 reagent (Invitrogen, Carlsbad, CA, USA) according to manufacturer's directions.

\section{Dual-luciferase reporter assay}

Dual-luciferase reporter assays were performed as previously described $[23,24]$. For promoter activity assay, the pGL3-basic vectors were co-transfected with pRL-TK as a control. Firefly and Renilla luciferase activities were measured at $48 \mathrm{~h}$ post-transfection using a Dual-GLO Luciferase Assay System Kit (Promega, Madison, WI, USA), following the manufacturer's instructions. Luminescence was measured by using a Fluorescence/MultiDetection Microplate Reader (BioTek, Winooski, VT, USA) and firefly luciferase activities were normalized to Renilla luminescence in each well.

\section{Chromatin immunoprecipitation (ChIP) assay}

ChIP assay was performed by using the ChIP assay kit (Beyotime, Shanghai, China) as recommended by the supplier. Chromatin was immunoprecipitated with the DYKDDDDK Tag (D6W5B) rabbit monoclonal antibody (14,793, 1:50, Cell Signaling Technology, Inc., Boston, 
USA). The relative quantity of the immunoprecipitated factor was calculated by qPCR.

\section{5-Ethynyl-2'-deoxyuridine (EdU), flow cytometry, and cell counting kit-8 (CCK-8) assay}

For the EdU assay, primary myoblasts seeded in 24-well plates were cultured to $50 \%$ density and then transfected. Forty-eight hours after transfection, the cells were fixed and stained with a C10310 EdU Apollo In Vitro Imaging Kit (RiboBio, China; $50 \mu \mathrm{mol} / \mathrm{L}$ ) as previously described [23]. A fluorescence microscope (DMi8; Leica, German) was used to capture three randomly selected fields to visualize the number of EdU-stained cells.

For the flow cytometry analysis of the cell cycle, myoblasts were seeded in 12 -well plates. After $48 \mathrm{~h}$ transfection, the cultured cells in growth media were collected and fixed overnight in $70 \%$ ethanol at $-20{ }^{\circ} \mathrm{C}$. Cells were analyzed by a BD AccuriC6 flow cytometer (BD Biosciences, San Jose, CA, USA) with the Cell Cycle Analysis Kit (Thermo Fisher Scientific, USA), and the data were processed using FlowJo software (7.6, Treestar Incorporated, Ashland, OR, USA).

For the CCK-8 assay, primary myoblasts were seeded in a 96-well plate and cultured in growth medium. After being transfected, the proliferation of the cell culture was monitored at $12 \mathrm{~h}, 24 \mathrm{~h}, 36 \mathrm{~h}$, and $48 \mathrm{~h}$ using the TransDetect CCK (TransGen Biotech, Beijing, China) as recommended by the supplier. The data of absorbance at $450 \mathrm{~nm}$ were read by an iMark $^{\mathrm{TM}}$ Microplate Absorbance Reader (Bio-Rad, California, USA).

\section{Immunoblotting and immunofluorescence (IF)}

Western blots were performed as previously described [20]. The primary antibodies used were anti-MyHC (B103, $0.5 \mu \mathrm{g} / \mathrm{mL}$, DHSB, Iowa City, IA, USA), antiMYOD (ABP53067, 1:500, Abbkine, Wuhan, China), anti-MYH1A (F59, $0.5 \mu \mathrm{g} / \mathrm{mL}$, DHSB, Iowa City, IA, USA), anti-MYH7B (S58, $0.5 \mu \mathrm{g} / \mathrm{mL}, \mathrm{DHSB}$, Iowa City, IA, USA), anti-NAMPT (bs-0272R, 1:500, Bioss, Beijing, China), anti-p-AMPK (ABN-PAB12602, 1:2000, Abnova, Taipei City, Taiwan, China), anti-AMPK (bs-1115R, 1: 500, Bioss, Beijing, China), anti-PGC1 $\alpha$ (66369-1-Ig, 1 : 5000, Proteintech, IL, USA), and anti- $\beta$-Tubulin (A01030, 1:10,000, Abbkine, Wuhan, China). ProteinFind Goat Anti-Mouse IgG $(\mathrm{H}+\mathrm{L})$, HRP Conjugate (HS201-01, 1:1000, TransGen, Beijing, China) and ProteinFind Goat Anti-Rabbit IgG $(\mathrm{H}+\mathrm{L})$, HRP Conjugate (HS101-01, 1:500, TransGen, Beijing, China) were used as a secondary antibody.

Immunofluorescence were performed using antiDesmin (bs-1026R, 1:100, Bioss, Beijing, China) and anti-MyHC (B103, $2.5 \mu \mathrm{g} / \mathrm{mL}, \mathrm{DHSB}$, Iowa City, IA, USA), as previously described [20]. A fluorescence microscope (DMi8; Leica, Germany) was used to capture three randomly selected fields to visualize the area labeled with anti-MyHC.

\section{Mitochondrial DNA (mtDNA) content assay}

Total DNA was extracted by using the Tissue DNA Kit (D3396, Omega, GA, USA) according to the manufacturer's instructions. The amount of mitochondrial DNA was determined by quantification of cytochrome c oxidase subunit II (COX2). The nuclear-encoded $\beta$-globin gene was used as internal controls. Primers used in this study can be found in the Table S1.

\section{Mitochondrial membrane potential and reactive oxygen species (ROS) concentration assay}

Mitochondrial membrane potential and ROS concentration were measured using the mitochondrial membrane potential assay kit with JC-1 (C2006, Beyotime, Shanghai, China) and reactive oxygen species assay kit (S0033S, Beyotime, Shanghai, China), according to the manufacturer's instructions.

\section{Enzyme activities assays}

The glycolytic capacity of myoblast was evaluated by the activity of lactic dehydrogenase (LDH), while the oxidative capacity of myoblast was evaluated by the activity of succinate dehydrogenase (SDH). Enzyme activities were measured by commercial assay kits (BC0685 and BC0955) that were purchased from Beijing Solarbio Science \& Technology.

\section{Biotin-coupled miRNA pull down assay}

The 3' end biotinylated miR-499-3p mimic and mimic $\mathrm{NC}$ were transfected into CPMs in T75 cell culture bottle. At $48 \mathrm{~h}$ after transfection, the cells were harvested and then lysed in lysis buffer. The biotin-coupled RNA complex was pull down, and then isolated as previously described [25]. The abundance of circPTPN4 and NAMPT in bound fractions was evaluated by quantitative PCR.

\section{Statistical analysis}

In this study, all experiments were repeated at least three times, and results were represented as mean \pm SEM. Where applicable, the statistical significance of the data was tested using independent sample $t$-test or ANOVA followed by Dunnett's test. The types of tests and the Pvalues, when applicable, are indicated in the figure legends.

\section{Results}

\section{Characterization of circRNAs in fast-twitch and slow-} twitch myofiber

In poultry, breast muscle is generally considered to be composed of fast-twitch myofibers, while the leg muscle has a higher proportion of slow-twitch fibers 
$[24,26]$. To systematically identify circRNAs involved in skeletal muscle development, we performed a circRNA-seq to analyze differentially expressed circRNAs between PEM (which is mainly composed of fast-twitch fibers) and SOL (which has higher proportion of slow muscle fibers) in 7-week-old Xinghua chicken. A total of 8882 circRNAs were detected, which were mainly (more than 85\%) distributed among chromosomes 1 to 15 , and W (Fig. 1A). According to their genomic locus, we found most of them $(\sim 75 \%)$ originate from coding exon (Fig. 1B). The length distribution of those circRNAs is relatively concentrated, with most in the range of $0-2000 \mathrm{nt}$ (Fig. 1C).

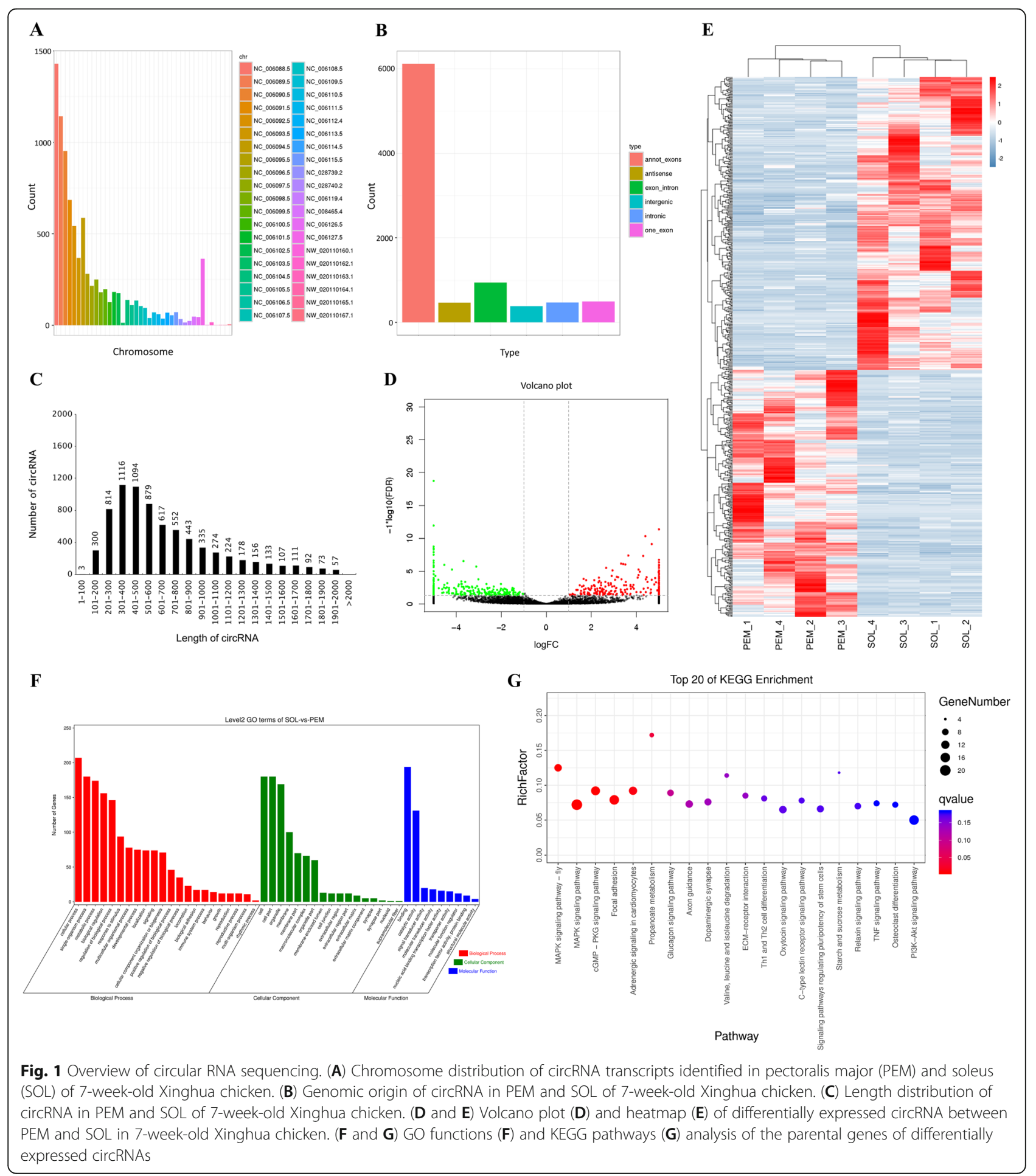


In total, 532 circRNAs were identified as being differentially expressed between PEM and SOL in 7-week-old Xinghua chicken $\left(P<0.05 ; \quad\left|\log _{2} \mathrm{FC}\right|>1\right) \quad$ (Table S3). Among the differentially expressed circRNAs, 243 showed upregulation in PEM, while 289 were increased in SOL (Fig. 1D and E; Table S3). Recent studies have found that the biogenesis of circRNA can competes with pre-mRNA splicing, and intron or exon-intron circRNAs

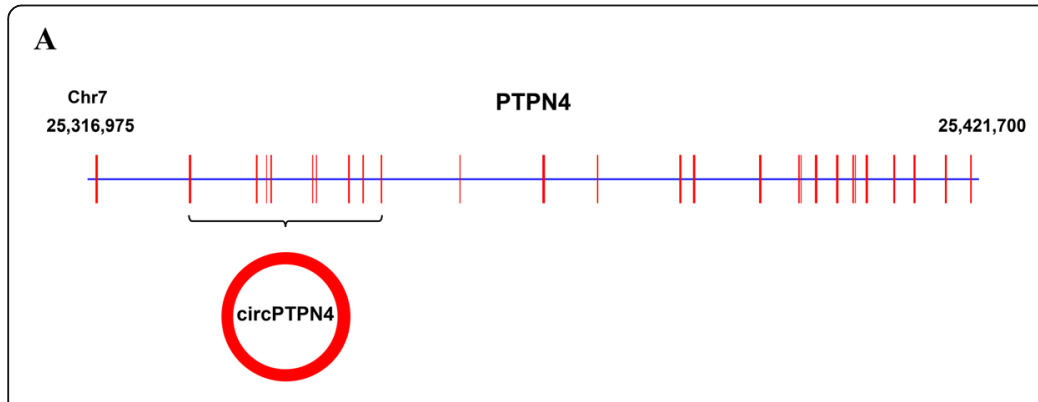

$\mathbf{B}$
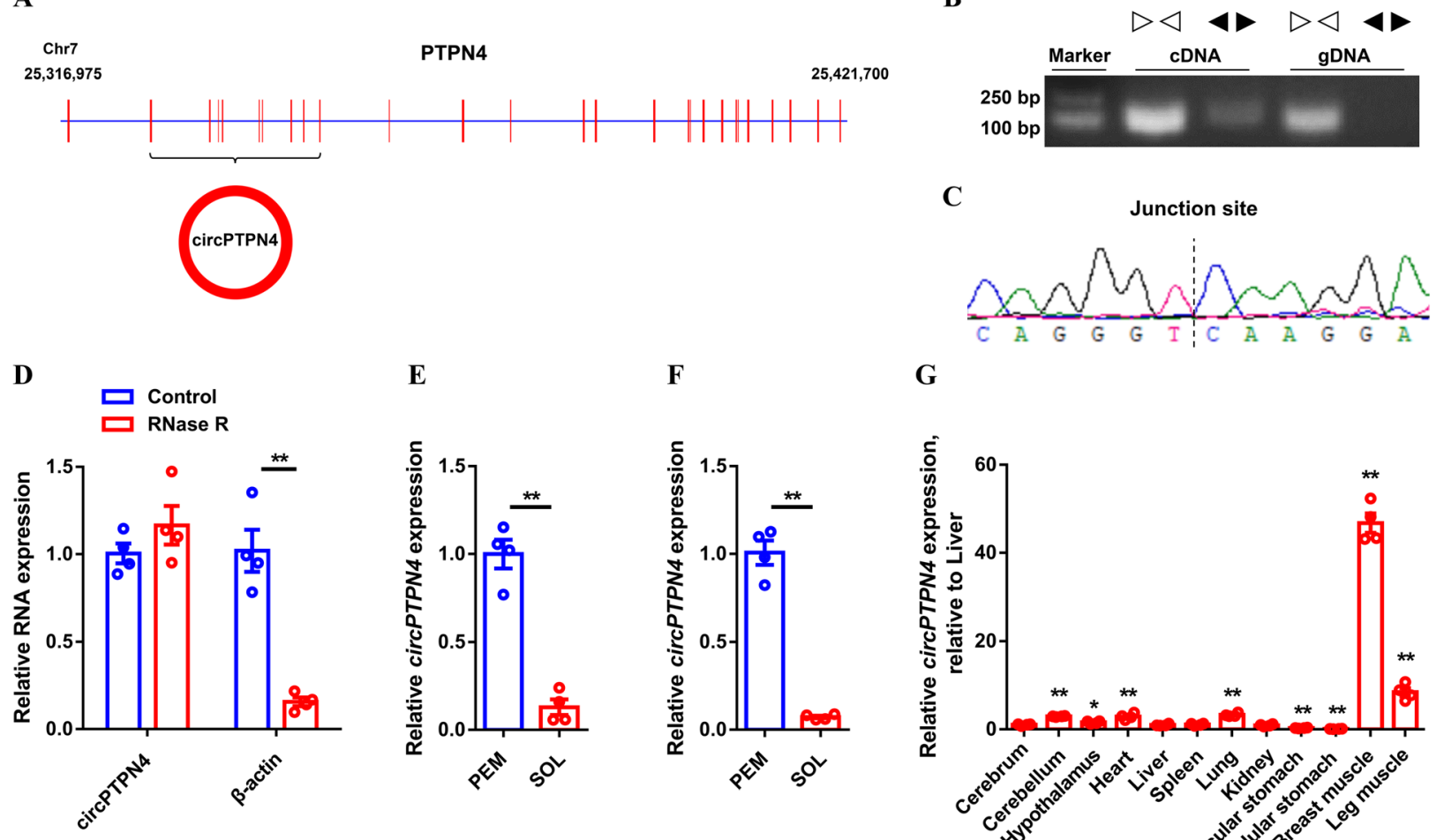

E

F

G
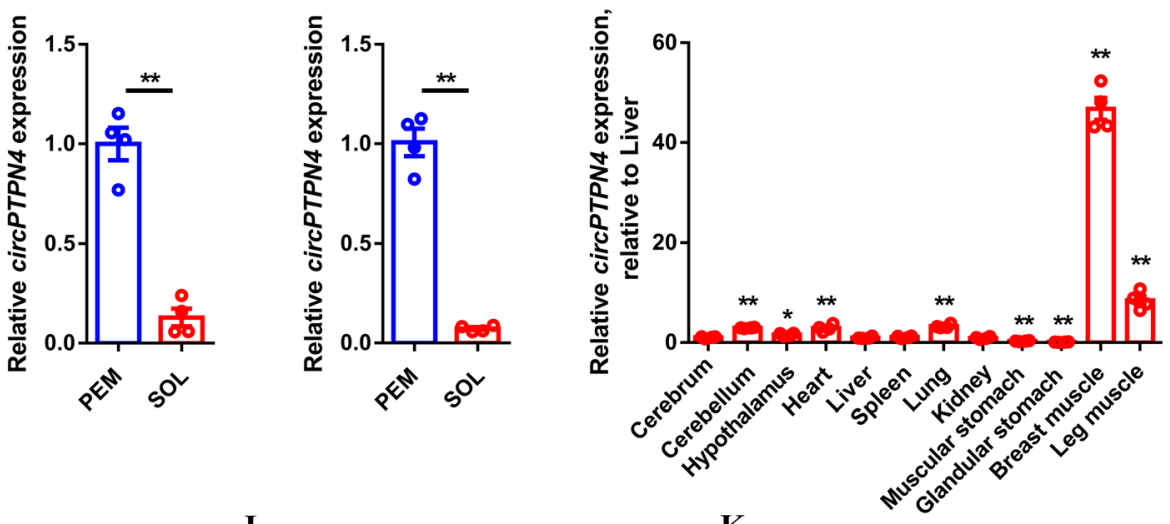

H

I

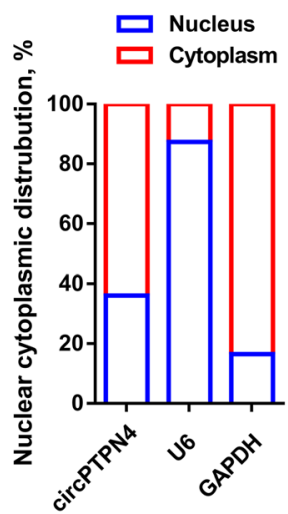

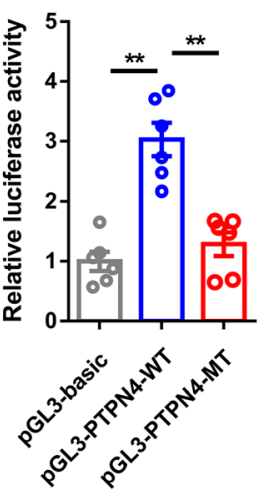

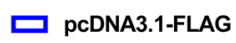
$\square$ pcDNA3.1-FOXA2-FLAG

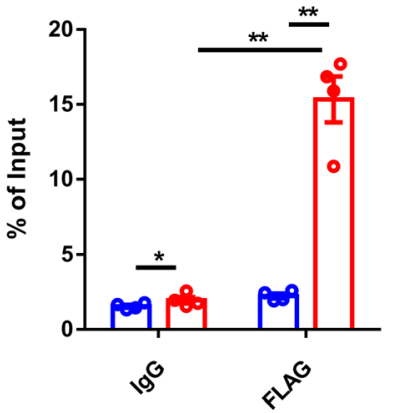

$\mathbf{K}$
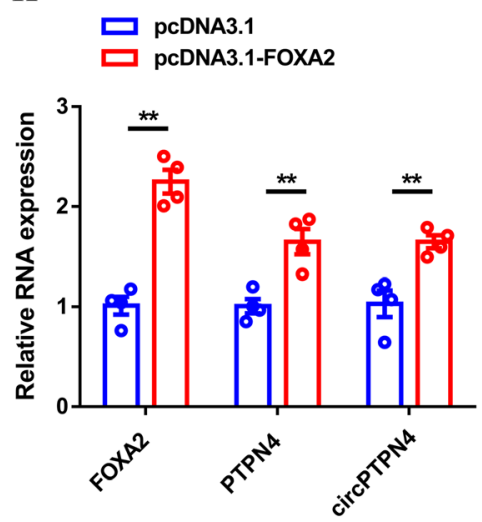

Fig. 2 Identification of circPTPN4. (A) Schematic image of circPTPN4 derived from PTPN4. (B) Verification of circPTPN4 by amplifying with divergent primers. (C) Sanger sequencing confirmed the back-splicing junction sequence of circPTPN4. (D) Relative circPTPN4 and $\beta$-actin expression after treatment with RNase R. (E and F) Relative circPTPN4 expression in pectoralis major (PEM) and soleus (SOL) of 7-week-old Xinghua chicken detected by RNA-seq $(\mathbf{E})$ and GPCR (F). (G) Tissue expression profiles of circPTPN4. The horizontal axis and vertical axis indicate different tissues and their relative expression values, respectively. $(\mathbf{H})$ The distribution of circPTPN4 in the cytoplasm and nuclei of chicken primary myoblast (CPM) was determined by qRT-PCR. GAPDH and U6 serve as cytoplasmic and nuclear localization controls, respectively. (I) The transcriptional activity of the PTPN4 core promoter region. (J) Chromatin immunoprecipitation (ChIP) analysis of the binding capacity of FOXA2 to the PTPN4 core promoter region. (K) Relative FOXA2, circPTPN4 and circPTPN4 expression with FOXA2 overexpression. Results are presented as mean \pm SEM. In panels ( $\mathbf{D}$ to $\mathbf{H}$, and $\mathbf{K}$ ), the statistical significance of differences between means was assessed using paired $t$-tests. In panels (I and $\mathbf{J})$, ANOVA followed by Dunnett's test was used. ( ${ }^{*} P<0.05$; $\left.{ }^{* *} P<0.01\right)$ 
can regulate the transcription of their parental gene [27-29]. Next, Gene Ontology (GO) and Kyoto Encyclopedia of Genes and Genomes (KEGG) enrichment analyses were performed for the parental genes of differentially expressed circRNAs. The results showed that these genes were mainly enriched in biological processes such as cellular process, metabolic process, and biological regulation, as well as participated in skeletal muscle development related pathways including MAPK signaling pathway, cGMP-PKG signaling pathway, PI3KAkt signaling pathway, and so on (Fig. 1F and G).
CircPTPN4 is a novel circRNA regulated by FOXA2

To further elucidate the regulation mechanism of circRNA involved in skeletal muscle development, a novel differentially expressed circRNA, circPTPN4 (novel_circ_ 002621; which was derived from exon 2-10 of PTPN4, highly conserved in Meleagris gallopavo, Numida meleagris and Anser cygnoides) (Fig. 2A and S2; Table S4), was served as a candidate. Firstly, genomic DNA (gDNA) and CDNA were used for the PCR reaction with convergent and divergent primers to confirm the sequence and the junction of $\operatorname{circPTPN4}$. A single distinct band with
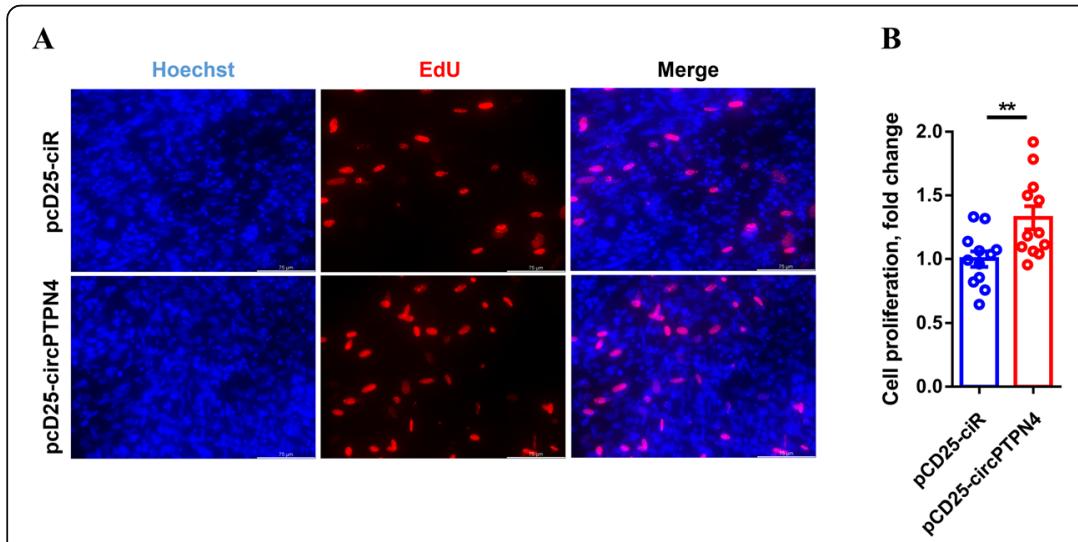

C 口 pCD25-ciR
pCD25-circPTPN4

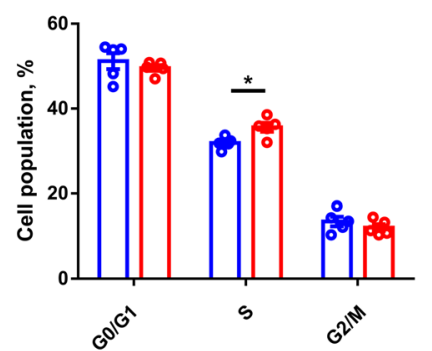

D

$\mathbf{E}$

$\mathbf{F}$

Hoechst

EdU

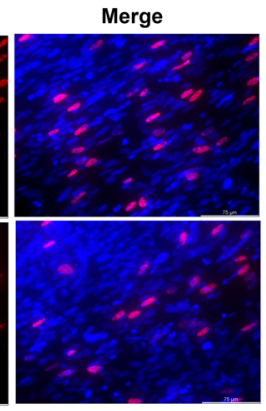

G

H

I
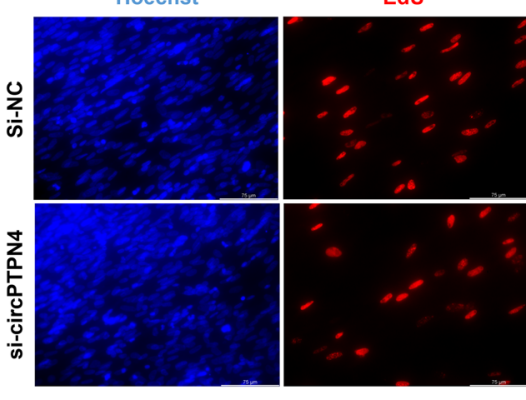

$\mathbf{J}$

- si-NC

口 si-NC

口 si-circPTPN4
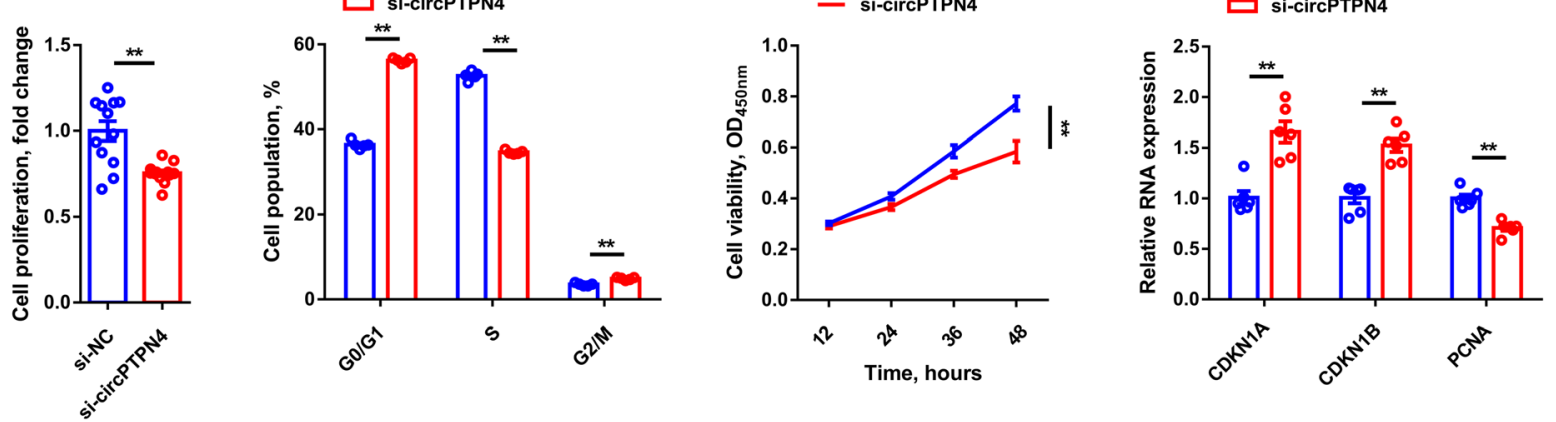

Fig. 3 circPTPN4 promotes myoblast proliferation. (A to E) EdU proliferation assay (A), the proliferation rate of myoblast (B), cell cycle analysis $(\mathbf{C})$, CCK-8 assay (D), and relative mRNA levels of several cell cycle genes $(\mathbf{E})$ with circPTPN4 overexpression in CPMs. (F to $\mathbf{J})$ EdU proliferation assay (F), proliferation rate of myoblast $(\mathbf{G})$, cell cycle analysis $(\mathbf{H})$, CCK-8 assay (I), and relative mRNA levels of several cell cycle genes $(\mathbf{J})$ after circPTPN4 interference in CPMs. Results are shown as mean \pm SEM. In panels (B to $\mathbf{E}$, and $\mathbf{G}$ to $\mathbf{J}$ ), the statistical significance of differences between means was assessed using independent sample $t$-test. $\left({ }^{*} P<0.05 ;{ }^{*} P<0.01\right)$ 
the expected product size was only observed in cDNA samples (Fig. 2B), and the real existence was detected by Sanger sequencing (Fig. 2C). These results suggested that the presence of back-splicing junctions but not genomic rearrangement. Moreover, the RNase R tolerance test showed $\operatorname{circPTPN} 4$ has more resistance than the linear mRNA control (Fig. 2D), which confirmed that circPTPN4 is a real circRNA. Our circRNA-seq data showed $\operatorname{circPTPN4}$ was differentially expressed between PEM and SOL in 7-week-old Xinghua chicken (Fig. 2E). Similarly, the consistent result was found by quantitative PCR (qPCR) (Fig. 2F). circPTPN4 was highly expressed in breast and leg muscle (Fig. 2G), implying that it may play an important role in skeletal muscle development.
In addition, cell-fractionation assays demonstrated that circPTPN4 is mainly present in the cytoplasm of chicken primary myoblast (CPM) (Fig. 2H).

To explore the mechanism through which $\operatorname{circPTPN4}$ is regulated at the transcriptional level, we further analyzed the core promoter region of PTPN4 (which is the parental gene of $\operatorname{circPTPN4}$ ), and found a potential binding site for FOXA2 ( -241 to $-228 \mathrm{bp}$ ). Dual-luciferase reporter assay confirmed that the mutation of this site leads to a decrease of the transcriptional activity (Fig. 2I and S3A), while the transcriptional activity was increased with FOXA2 overexpression (Fig. S3B). Moreover, results of a chromatin immunoprecipitation (ChIP) assay confirmed that FOXA2 could physically bind to the core

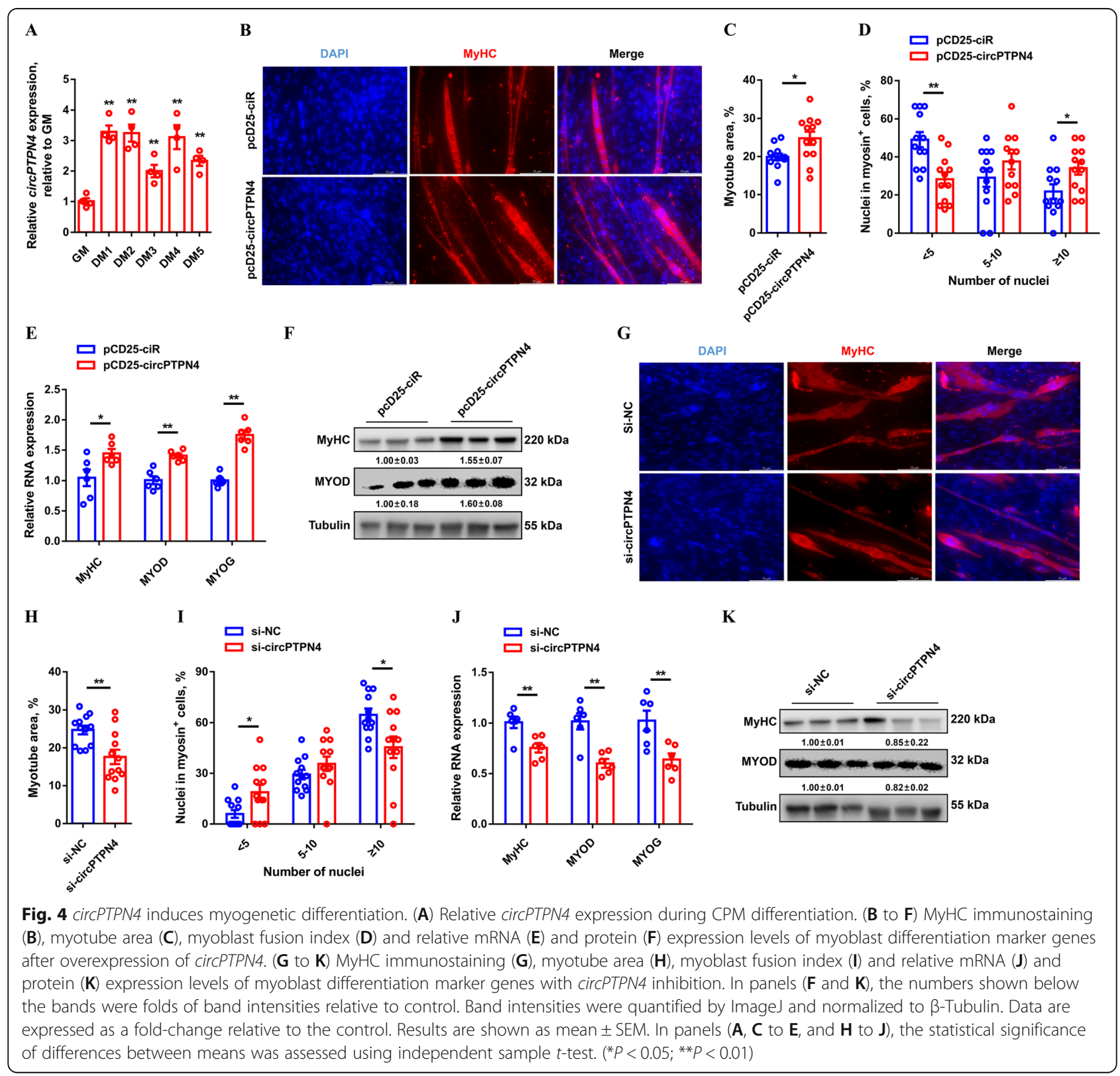




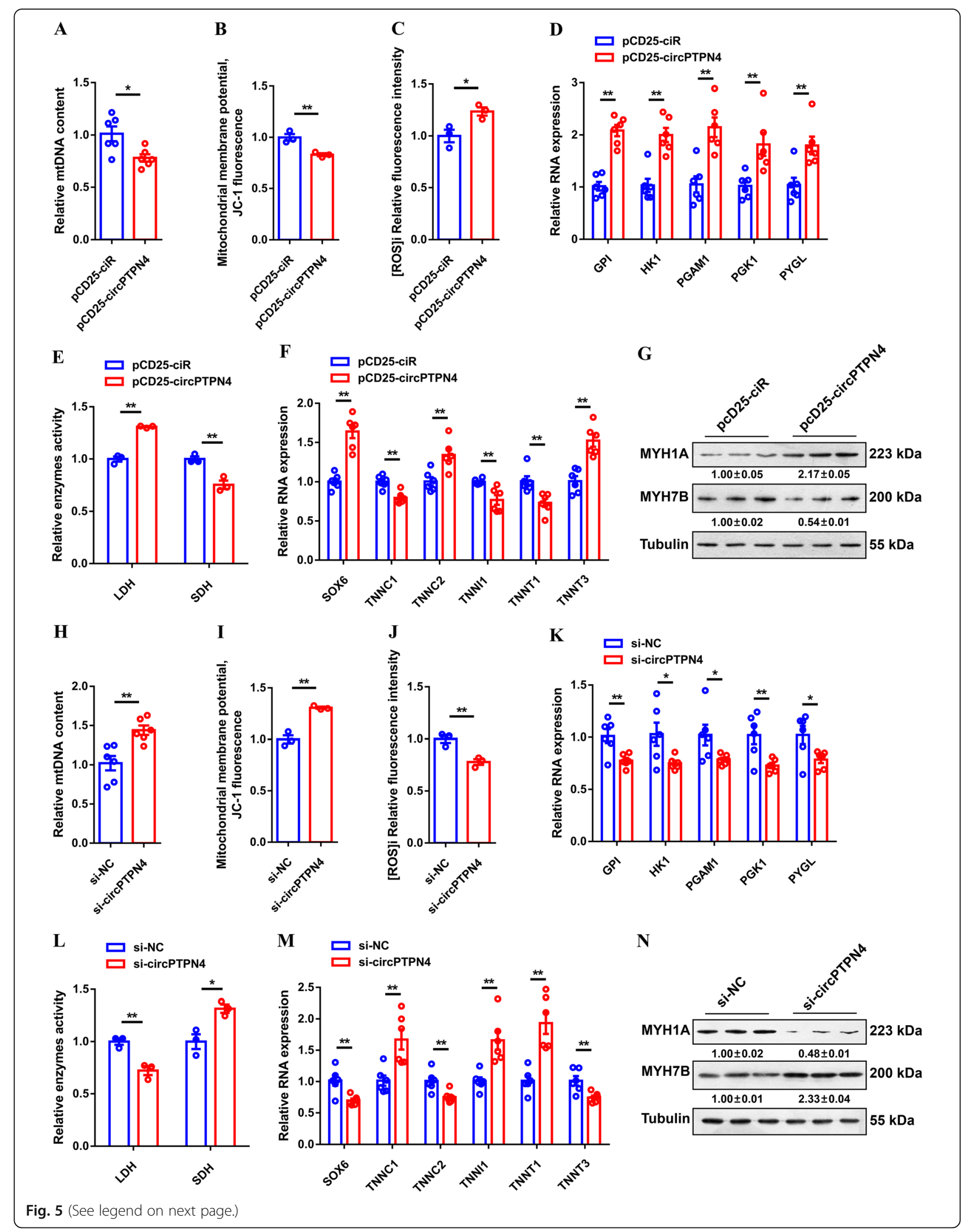


(See figure on previous page.)

Fig. 5 circPTPN4 represses mitochondria biogenesis and drives the transformation of slow-twitch to fast-twitch myofiber. (A to G) Relative mitochondrial DNA (mtDNA) content $(\mathbf{A})$, mitochondrial membrane potential $(\mathbf{B})$, reactive oxygen species (ROS) production $(\mathbf{C})$, relative mRNA expression levels of glycogenolytic and glycolytic genes (D), relative enzymes activity of lactic dehydrogenase (LDH) and succinate dehydrogenase $(\mathrm{SDH})(\mathbf{E})$, relative mRNA expression levels of several fast-/slow-twitch myofiber genes $(\mathbf{F})$, and relative protein expression of MYH1A and MYH7B (G) in circPTPN4 overexpression CPMs. ( $\mathbf{H}$ to $\mathbf{N})$ Relative mtDNA content (H), mitochondrial membrane potential (I), ROS production $(\mathbf{J})$, relative mRNA expression levels of glycogenolytic and glycolytic genes (K), relative enzymes activity of LDH and SDH (L), relative mRNA expression levels of several fast-/slow-twitch myofiber genes (M), and relative protein expression of MYH1A and MYH7B (N) in CPMs with cirCPTPN4 interference. In panels ( $\mathbf{G}$ and $\mathbf{N}$ ), the numbers shown below the bands were folds of band intensities relative to control. Band intensities were quantified by ImageJ and normalized to $\beta$-Tubulin. Data are expressed as a fold-change relative to the control. In all panels, results are shown as mean \pm SEM, the statistical significance of differences between means was assessed using independent sample $t$-test. $\left({ }^{*} P<0.05 ;{ }^{* *} P<0.01\right)$

promoter of PTPN4 (Fig. 2J). Overexpression of FOXA2 upregulated the expression of PTPN4 and circPTPN4 (Fig. 2K). Collectively, these data revealed that circPTPN4 is positively regulated by the FOXA2.

\section{circPTPN4 facilitates the proliferation and differentiation of myoblast}

In order to assess the function of $\operatorname{circPTPN} 4$ in myogenesis, the overexpression vector of circPTPN4 was constructed and transfected into CPM (Fig. S4A). The 5ethynyl-2'-deoxyuridine (EdU) staining demonstrated that circPTPN4 overexpression significantly increased EdU incorporation and promoted myoblast proliferation (Fig. 3A and B). Flow cytometric analysis and cell counting kit-8 (CCK-8) assay also showed that overexpression of circPTPN4 significantly increased the number of $\mathrm{S}$ phase cells (Fig. 3C), and improved myoblast viability (Fig. 3D). Furthermore, circPTPN4 overexpression repressed the expression level of cell cycle-inhibiting genes, including $C D K N 1 A$ and $C D K N 1 B$, while increasing the expression level of cell cycle-promoting genes like PCNA (Fig. 3E). Conversely, the opposite result was observed by circPTPN4 interference (Fig. 3F to J, and S4B), indicating that circPTPN4 can facilitate myoblast proliferation.

circPTPN4 expression was upregulated with myogenic differentiation (Fig. 4A), which suggested that $\operatorname{circPTPN4}$ may be involved in the process of myoblast differentiation. To further investigate the potential function of circPTPN4, immunofluorescence staining was performed. Immunofluorescence staining showed that overexpression of circPTPN4 increased the total areas of myotubes and induced myotube formation (Fig. 4B to D). In addition, the expressions level of myoblast differentiation marker genes, including $M y H C, M Y O D$, and MYOG were significantly upregulated with $\operatorname{circPTPN4}$ overexpression (Fig. $4 \mathrm{E}$ and F). On the contrary, circPTPN4 interference decreased the total areas of myotubes and inhibited myoblast fusion, as well as downregulated the expression of myoblast differentiation marker genes (Fig. 4G to $\mathrm{K}$ ).

\section{circPTPN4 suppresses mitochondria biogenesis and activates fast-twitch muscle phenotype}

Skeletal muscle is a major player in regulating energy homeostasis [30, 31]. As the main organelle of energy metabolism, mitochondria are closely related to the development of skeletal muscle [32, 33]. Next, we evaluated mitochondrial content and function after overexpression and inhibition of $\operatorname{circPTPN} 4$. circPTPN4 overexpression decreased mitochondrial DNA (mtDNA) content and was accompanied by a decline of mitochondrial membrane potential (Fig. 5A and B). Meanwhile, reactive oxygen species (ROS) production was significantly increased after circPTPN4 overexpression (Fig. 5C). Inversely, circPTPN4 inhibition increased mitochondrial content and enhanced mitochondrial function (Fig. $5 \mathrm{H}$ to $\mathrm{J}$ ), illustrating that circPTPN4 suppresses mitochondria biogenesis.

Skeletal muscle is comprised of heterogeneous myofibers that differ in their physiological and metabolic parameters [34]. Compared with slow-twitch (type I; oxidative) myofibers, fast-twitch (type II; glycolytic) myofibers have fewer mitochondria and higher activity of glycolytic metabolic enzymes [35, 36]. Given that circPTPN4 is highly expressed in fast-twitch myofiber and repressed mitochondria biogenesis, we speculated that circPTPN4 may function in the activization of fast-twitch muscle phenotype. As expected, overexpression of circPTPN4 upregulated the expression of glycogenolytic and glycolytic genes (Fig. 5D). The activity of lactate dehydrogenase (LDH) was enhanced, while the activity of succinate dehydrogenase (SDH) was suppressed with circPTPN4 overexpression (Fig. 5E). circPTPN4 overexpression upregulated expressions of multiple fast-twitch myofiber genes like SOX6, TNNC2 and TNNT3, while suppressed slowtwitch myofiber genes such as TNNC1, TNNI1 and TNNT1 (Fig. 5F). More importantly, western blot results showed that overexpression of circPTPN4 promoted MYH1A/fast-twitch protein level and suppressed the expression level of $\mathrm{MYH7B} / \mathrm{slow}$ twitch protein (Fig. 5G). On the contrary, the glycolytic capacity of skeletal muscle was suppressed and 
the slow-twitch muscle phenotype was induced with circPTPN4 interference (Fig. $5 \mathrm{~K}$ to $\mathrm{N}$ ).

circPTPN4 interacts with miR-449-3p to upregulate NAMPT expression, thus inactivating AMPK signaling

In 2011, competitive endogenous RNAs (ceRNAs) were first reported as endogenous sponges that can affect the distribution of miRNAs on their targets, thereby imposing another novel layer of posttranscriptional regulation
[37]. Given that circPTPN4 is mainly present in the cytoplasm, we hypothesized that circPTPN4 may function as a ceRNA to exert its biological function. The target miRNAs and genes of circPTPN4 were predicted on an RNAhybrid software. Interestingly, $m i R-499-3 p$ was found to contain potential binding sites for both circPTPN4 and NAMPT (Fig. 6A), suggesting that it may mediate the regulation of $N A M P T$ expression by circPTPN4. Compared with PEM, the expression of $m i R-$

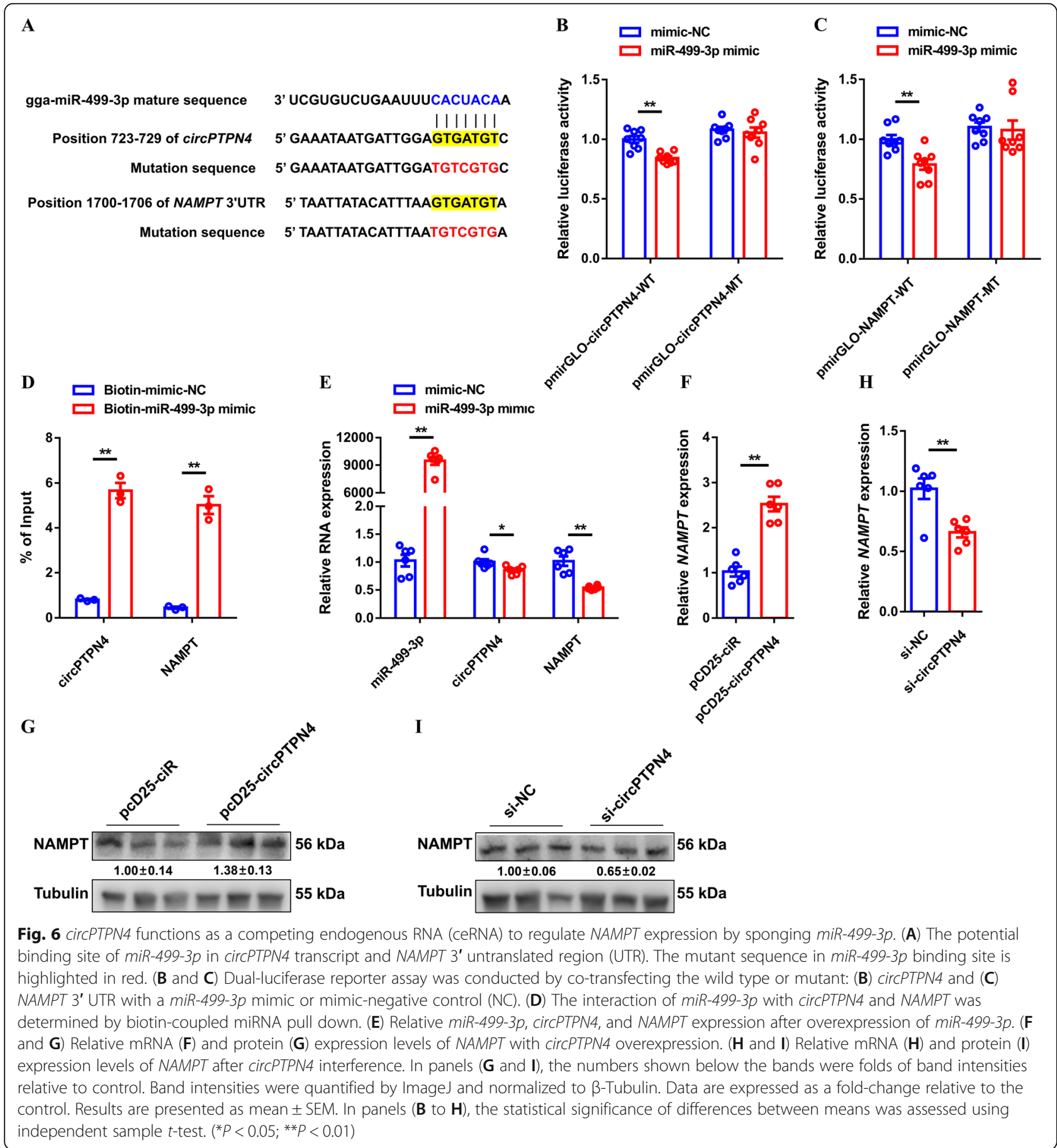




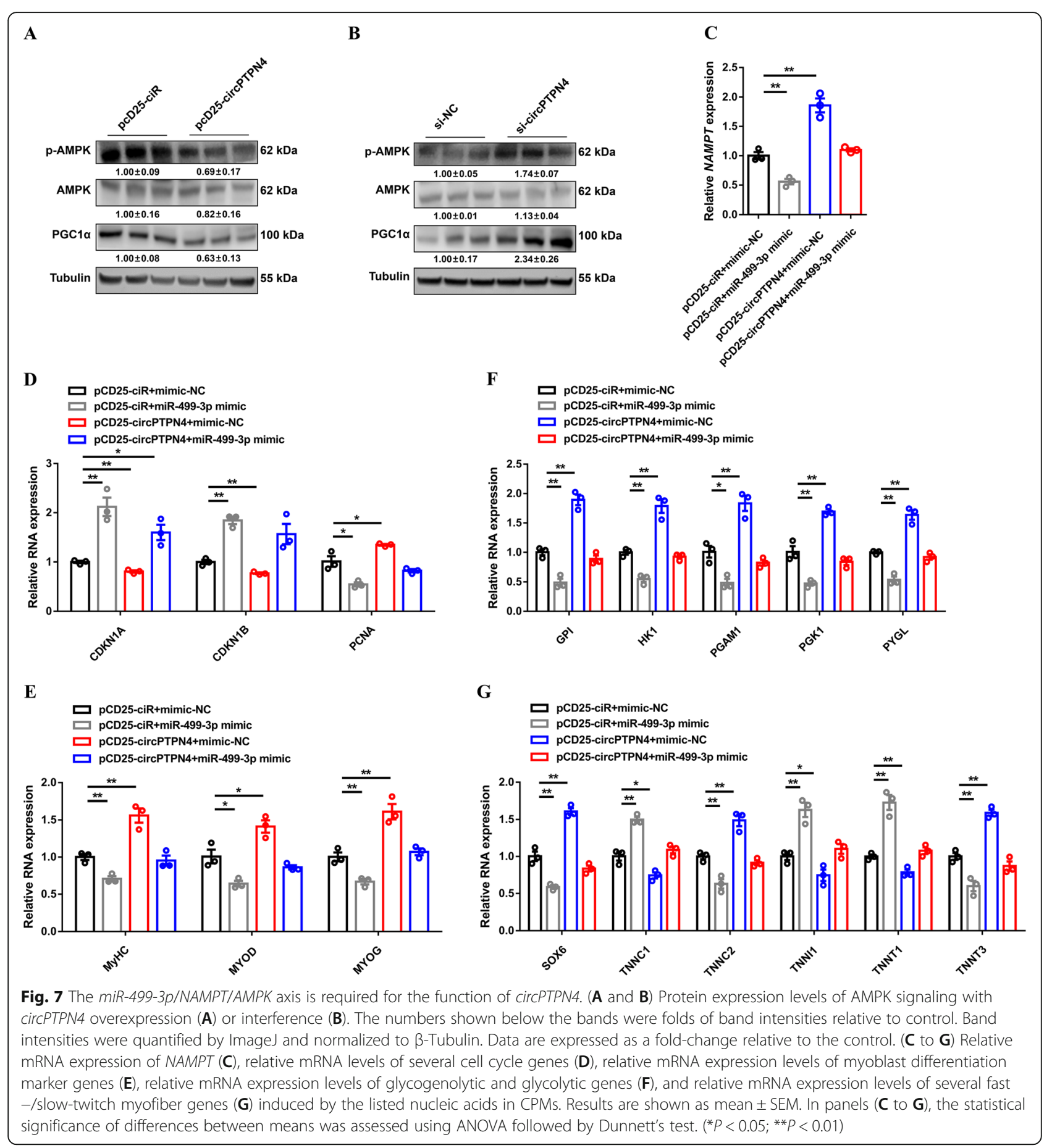

499-3 $p$ is higher in SOL (Fig. S5A). In contrast, NAMPT is highly expressed in PEM (Fig. S5B), which is consistent with circPTPN4, further hinting that the interaction of circPTPN4 with miR-499-3p and NAMPT. Dualluciferase reporter assays were carried out to confirm whether miR-499-3p directly interact with circPTPN4 and NAMPT. The results showed that miR-499-3p bind with both $\operatorname{circPTPN4}$ and the 3' UTR of NAMPT (Fig. $6 \mathrm{~B}$ to $\mathrm{C})$. Furthermore, the interaction of $m i R-499-3 p$ with circPTPN4 and NAMPT was also verified by a biotin-coupled miRNA pull down assay (Fig. 6D). Overexpression of $m i R-499-3 p$ repressed the expression of circPTPN4 and NAMPT (Fig. 6E). More importantly, circPTPN4 overexpression upregulated the mRNA and protein levels of $N A M P T$, whereas the expression of $N A M P T$ was suppressed with $\operatorname{circPTPN4}$ interference (Fig. 6F-I), explaining the targeted regulation of circPTPN4 on NAMPT. 
Previous studies have shown that NAMPT is widely involved in a series of biological processes by inactivating AMPK signaling [38-40]. We further assessed the AMPK signaling and found that circPTPN4 overexpression inhibited the phosphorylation of AMPK and downregulated the expression of PGC1 $\alpha$ (Fig. 7A). Conversely, this pathway was activated with the interference of $\operatorname{circPTPN4}$ (Fig. 7B), suggesting that $\operatorname{circPTPN4}$ may participate in AMPK signaling by regulating $N A M P$ $T$. Overexpression of $\operatorname{circPTPN} 4$ alleviated the inhibitory effect of $m i R-499-3 p$ on NAMPT expression (Fig. 7C). In addition, the regulatory effects of $\operatorname{circPTPN4}$ were weakened after $m i R-499-3 p$ overexpression (Fig. 7D to G), indicating that the $m i R-499-3 p / N A M P T / A M P K$ axis is required for the function of $\operatorname{circPTPN4}$.

\section{Discussion}

Due to the low quantity and expressive abundance, circRNAs were once considered to be an abnormal splicing product of RNA or unique structure of pathogens, with less attention [41, 42]. However, recent studies have found that circRNA is universally present in archaea, suggesting that it may have important biological functions [43]. With the development of genome research, more and more circRNAs are found in various cells and tissues, which are widely present in eukaryotes [44-47]. In this study, a total of 532 circRNAs were identified as being differentially expressed between PEM and SOL in 7-week-old Xinghua chicken. Among them, a novel differentially expressed circRNA, circPTPN4, was served as a candidate. circPTPN4 is highly expressed in fast-twitch myofiber, and its expression upregulates with myoblast differentiation, suggesting that it may play a significant role in skeletal muscle development.

Myogenesis is a process including myoblast proliferation, differentiation and myotube formation and is controlled by a series of myogenic regulatory factors. These factors can regulate myoblasts to withdraw from the cell cycle, express muscle-specific genes, and prevent the expression of other cell- or tissue-specific genes. Recently, it is worth noting that circRNAs have also been demonstrated to function in myogenesis [48-51]. Here, we found that $\operatorname{circPTPN} 4$ promotes myoblast proliferation and induces myogenic differentiation.

Skeletal muscle is composed of different types of myofibers. Under certain conditions, different types of myofibers can be transformed. Previous studies have found that a total of 305 circRNAs were differentially expressed between the oxidative muscle sartorius compared and the glycolytic muscle pectoralis major in Chinese Qingyuan partridge chickens. Among them, novel_circ_ 004282 and novel_circ_002121 were speculated to play important roles in regulating oxidative myofibers by PPP3CA and NFATC1 expression [52]. As a transcriptional coactivator, $\mathrm{PGC} 1 \alpha$ is a downstream effector of AMPK signaling, has been found to regulate mitochondria biogenesis and the transformation of myofiber type [53-55]. In the current study, we found that circPTPN4 decreases mtDNA content and suppresses mitochondria functions. Moreover, $\operatorname{circPTPN} 4$ improves the glycolytic capacity of myoblast to activate fast-twitch muscle phenotype, demonstrating that circPTPN4 is involved in the transformation of myofiber type by inactivating AMPK signaling.

Recently, a new pattern of gene expression has been come up with regarding the interaction of RNA transcripts, called ceRNA [37]. There is a great deal of researches indicated that circRNAs can function as ceRNAs to protect mRNAs by acting as molecular sponges for miRNAs, thereby modulating the derepression of miRNA targets and imposing an additional level of post-transcriptional regulation $[46,49,50]$. In this study, using in silico analysis, we found $m i R-499-3 p$ contains binding sites for circPTPN4 and NAMPT. The interaction of $m i R-499-3 p$ with $\operatorname{circPTPN} 4$ and NAMPT was further validated by dual-luciferase reporter assay and biotin-coupled miRNA pull down assay. circPTPN4

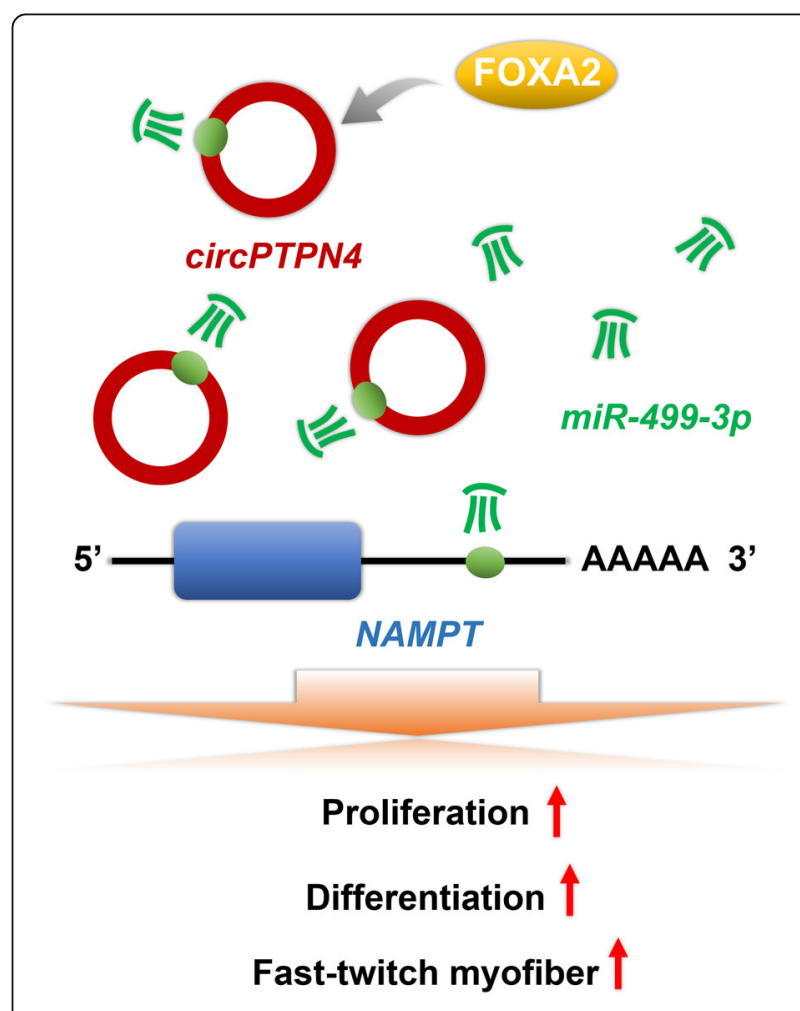

Fig. 8 Model of circPTPN4 functions as a ceRNA to regulate NAMPT expression by sponging miR-499-3p, thus promoting the proliferation and differentiation of myoblast, as well as activating fast-twitch muscle phenotype 
regulates NAMPT expression to function in AMPK signaling. In addition, our rescue experiment showed that the biological functions of $\operatorname{circPTPN} 4$ were weakened with miR-499-3p overexpression, explaining that the $m i R-499-3 p / N A M P T$ axis is required for the function of circPTPN4.

\section{Conclusions}

In conclusion, we demonstrated that circPTPN4 is a novel circRNA, which is highly expressed in fast-twitch myofiber and is positively regulated by transcription factor FOXA2. Mechanistically, $\operatorname{circPTPN} 4$ can function as a ceRNA to regulate NAMPT expression by sponging $m i R-499-3 p$, thus promoting the proliferation and differentiation of myoblast, as well as activating fast-twitch muscle phenotype (Fig. 8). Our findings provide a solid foundation for the understanding of the mechanisms and regulatory networks of myogenesis, and will contribute to the development of further research.

\section{Abbreviations}

CCK-8: Cell counting kit-8; ceRNA: Competing endogenous RNA; ChIP: Chromatin immunoprecipitation; circRNA: Circular RNA; CPMs: Chicken primary myoblasts; EdU: 5-Ethynyl-2'-deoxyuridine; gDNA: Genomic DNA; GO: Gene Ontology; IF: Immunofluorescence; KEGG: Kyoto Encyclopedia of Genes and Genomes; LDH: Lactic dehydrogenase; miRNA: MicroRNA; mtDNA: Mitochondrial DNA; NAMPT: Nicotinamide phosphoribosyltransferase; NC: Negative control; ncRNA: Noncoding RNA; PEM: Pectoralis major; ROS: Reactive oxygen species; SDH: Succinate dehydrogenase; siRNA: Small interfering RNA; SOL: Soleus; UTR: Untranslated region

\section{Supplementary Information}

The online version contains supplementary material available at https://doi. org/10.1186/s40104-021-00664-1.

\section{Additional file 1.}

\section{Acknowledgements}

Not applicable.

\section{Authors' contributions}

QN and XZ conceived and designed the study. BC and MM performed the experiments, interpreted the data and wrote the paper. ZZ, SK and JZ performed the experiments. All authors read and approved the final manuscript.

\section{Funding}

This work was supported by Local Innovative and Research Teams Project of Guangdong Province (2019BT02N630), the Natural Scientific Foundation of China (U1901206 and 31761143014), Guangzhou Science and Technology Key Project (202103000084), and China Agriculture Research System (CARS41-G03).

\section{Availability of data and materials}

The data were exhibited in the main manuscript and supplemental materials.

\section{Declarations}

Ethics approval and consent to participate

All animal experimental protocols were conformed to "The Instructive Notions with Respect to Caring for Laboratory Animals" issued by the Ministry of Science and Technology of the People's Republic of China, and approved by the Institutional Animal Care and Use Committee at the South China Agricultural University (approval ID: 2021-C018).

\section{Consent for publication}

Not applicable.

\section{Competing interests}

The authors declare that they have no conflict of interest.

Received: 10 August 2021 Accepted: 2 December 2021

Published online: 14 February 2022

\section{References}

1. Lee SH, Joo ST, Ryu YC. Skeletal muscle fiber type and myofibrillar proteins in relation to meat quality. Meat Sci. 2010;86(1):166-70. https://doi.org/10.1 016/j.meatsci.2010.04.040.

2. Joo ST, Kim GD, Hwang YH, Ryu YC. Control of fresh meat quality through manipulation of muscle fiber characteristics. Meat Sci. 2013;95(4):828-36. https://doi.org/10.1016/j.meatsci.2013.04.044.

3. Wilkins MR, Sanchez JC, Gooley AA, Appel RD, Humphery-Smith I, Hochstrasser DF, et al. Progress with proteome projects: why all proteins expressed by a genome should be identified and how to do it. Biotechnol Genet Eng Rev. 1996;13(1):19-50. https://doi.org/10.1080/02648725.1996.1 0647923.

4. Djebali S, Davis CA, Merkel A, Dobin A, Lassmann T, Mortazavi A, et al. Landscape of transcription in human cells. Nature. 2012;489(7414):101-8. https://doi.org/10.1038/nature11233.

5. Xu M, Chen X, Chen D, Yu B, Li M, He J, et al. Regulation of skeletal myogenesis by microRNAs. J Cell Physiol. 2020;235(1):87-104. https://doi. org/10.1002/jcp.28986.

6. Chen R, Lei S, Jiang T, Zeng J, Zhou S, She Y. Roles of IncRNAs and circRNAs in regulating skeletal muscle development. Acta Physiol (Oxford). 2020; 228(2):e13356. https://doi.org/10.1111/apha.13356.

7. Memczak S, Jens M, Elefsinioti A, Torti F, Krueger J, Rybak A, et al. Circular RNAs are a large class of animal RNAs with regulatory potency. Nature. 2013;495(7441):333-8. https://doi.org/10.1038/nature11928.

8. Rybak-Wolf A, Stottmeister C, Glazar P, Jens M, Pino N, Giusti S, et al. Circular RNAs in the mammalian brain are highly abundant, conserved, and dynamically expressed. Mol Cell. 2015;58(5):870-85. https://doi.org/10.1016/j. molcel.2015.03.027.

9. Chen LL. The biogenesis and emerging roles of circular RNAs. Nat Rev Mol Cell Biol. 2016;17(4):205-11. https://doi.org/10.1038/nrm.2015.32.

10. Dong R, Zhang XO, Zhang Y, Ma XK, Chen LL, Yang L. CircRNA-derived pseudogenes. Cell Res. 2016;26(6):747-50. https://doi.org/10.1038/cr.2016.42.

11. Bach DH, Lee SK, Sood AK. Circular RNAs in cancer. Mol Ther Nucleic Acids. 2019;16:118-29. https://doi.org/10.1016/j.omtn.2019.02.005.

12. Wu J, Qi X, Liu L, Hu X, Liu J, Yang J, et al. Emerging epigenetic regulation of circular RNAs in human cancer. Mol Ther Nucleic Acids. 2019;16:589-96. https://doi.org/10.1016/j.omtn.2019.04.011.

13. Bartel DP. MicroRNAs: genomics, biogenesis, mechanism, and function. Cell. 2004;116(2):281-97. https://doi.org/10.1016/s0092-8674(04)00045-5.

14. Wahid F, Shehzad A, Khan T, Kim YY. MicroRNAs: synthesis, mechanism, function, and recent clinical trials. Biochim Biophys Acta. 2010;1803(11): 1231-43. https://doi.org/10.1016/j.bbamcr.2010.06.013.

15. Liu X, Zhang Y, Liang H, Zhang Y, Xu Y. MicroRNA-499-3p inhibits proliferation and promotes apoptosis of retinal cells in diabetic retinopathy through activation of the TLR4 signaling pathway by targeting IFNA2. Gene. 2020;741:144539. https://doi.org/10.1016/j.gene.2020.144539.

16. Jiang $A$, Yin D, Zhang L, Li B, Li R, Zhang $X$, et al. Parsing the microRNA genetics basis regulating skeletal muscle fiber types and meat quality traits in pigs. Anim Genet. 2021;52(3):292-303. https://doi.org/10.1111/age.13064.

17. Samal B, Sun Y, Stearns G, Xie C, Suggs S, McNiece I. Cloning and characterization of the cDNA encoding a novel human pre-B-cell colonyenhancing factor. Mol Cell Biol. 1994;14(2):1431-7. https://doi.org/10.1128/ mcb.14.2.1431-1437.1994.

18. Imai S. Nicotinamide phosphoribosyltransferase (Nampt): a link between NAD biology, metabolism, and diseases. Curr Pharm Des. 2009;15(1):20-8. https://doi.org/10.2174/138161209787185814.

19. Garten A, Schuster S, Penke M, Gorski T, de Giorgis T, Kiess W. Physiological and pathophysiological roles of NAMPT and NAD metabolism. Nat Rev Endocrinol. 2015;11(9):535-46. https://doi.org/10.1038/nrendo.2015.117. 
20. Cai B, Ma M, Chen B, Li Z, Abdalla BA, Nie Q, et al. MiR-16-5p targets SESN to regulate the p53 signaling pathway, affecting myoblast proliferation and apoptosis, and is involved in myoblast differentiation. Cell Death Dis. 2018; 9(3):367. https://doi.org/10.1038/s41419-018-0403-6.

21. Jeck WR, Sorrentino JA, Wang K, Slevin MK, Burd CE, Liu J, et al. Circular RNAs are abundant, conserved, and associated with ALU repeats. RNA. 2013; 19(2):141-57. https://doi.org/10.1261/rna.035667.112.

22. Cai B, Li Z, Ma M, Zhang J, Kong S, Abdalla BA, et al. Long noncoding RNA SMUL suppresses SMURF2 production-mediated muscle atrophy via nonsense-mediated mRNA decay. Mol Ther Nucleic Acids. 2021;23:512-26. https://doi.org/10.1016/j.omtn.2020.12.003.

23. Cai B, Li Z, Ma M, Wang Z, Han P, Abdalla BA, et al. LncRNA-Six1 encodes a micropeptide to activate six 1 in cis and is involved in cell proliferation and muscle growth. Front Physiol. 2017;8:230. https://doi.org/10.3389/fphys.2017. 00230.

24. Ma M, Cai B, Jiang L, Abdalla BA, Li Z, Nie Q, et al. LnCRNA-Six1 is a target of miR-1611 that functions as a ceRNA to regulate six1 protein expression and fiber type switching in chicken myogenesis. Cells. 2018;7(12):243. https://doi. org/10.3390/cells7120243.

25. Orom UA, Lund AH. Isolation of microRNA targets using biotinylated synthetic microRNAs. Methods. 2007;43(2):162-5. https://doi.org/10.1016/j. ymeth.2007.04.007

26. Li L, Liu HH, Xu F, Si JM, Jia J, Wang JW. MyoD expression profile and developmental differences of leg and breast muscle in Peking duck (Anas platyrhynchos Domestica) during embryonic to neonatal stages. Micron. 2010;41(7):847-52. https://doi.org/10.1016/j.micron.2010.04.016.

27. Zhang $Y$, Zhang $X O$, Chen $T$, Xiang JF, Yin QF, Xing YH, et al. Circular intronic long noncoding RNAs. Mol Cell. 2013;51(6):792-806. https://doi. org/10.1016/j.molcel.2013.08.017.

28. Ashwal-Fluss R, Meyer M, Pamudurti NR, Ivanov A, Bartok O, Hanan M, et al. CircRNA biogenesis competes with pre-mRNA splicing. Mol Cell. 2014;56(1): 55-66. https://doi.org/10.1016/j.molcel.2014.08.019.

29. Li Z, Huang C, Bao C, Chen L, Lin M, Wang X, et al. Exon-intron circular RNAs regulate transcription in the nucleus. Nat Struct Mol Biol. 2015;22(3): 256-64. https://doi.org/10.1038/nsmb.2959.

30. Zurlo F, Larson K, Bogardus C, Ravussin E. Skeletal muscle metabolism is a major determinant of resting energy expenditure. J Clin Invest. 1990;86(5): 1423-7. https://doi.org/10.1172/JCl114857.

31. Ibrahim A, Neinast M, Arany ZP. Myobolites: muscle-derived metabolites with paracrine and systemic effects. Curr Opin Pharmacol. 2017;34:15-20. https://doi.org/10.1016/j.coph.2017.03.007.

32. Boengler K, Kosiol M, Mayr M, Schulz R, Rohrbach S. Mitochondria and ageing: role in heart, skeletal muscle and adipose tissue. J Cachexia Sarcopenia Muscle. 2017;8(3):349-69. https://doi.org/10.1002/jcsm.12178.

33. Boncompagni S, Pozzer D, Viscomi C, Ferreiro A, Zito E. Physical and functional cross talk between Endo-sarcoplasmic reticulum and mitochondria in skeletal muscle. Antioxid Redox Signal. 2020;32(12):873-83. https://doi.org/10.1089/ars.2019.7934.

34. Bassel-Duby R, Olson EN. Signaling pathways in skeletal muscle remodeling. Annu Rev Biochem. 2006;75(1):19-37. https://doi.org/10.1146/annurev. biochem.75.103004.142622.

35. Schiaffino S, Reggiani C. Fiber types in mammalian skeletal muscles. Physiol Rev. 2011;91(4):1447-531. https://doi.org/10.1152/physrev.00031.2010.

36. Koutakis P, Weiss DJ, Miserlis D, Shostrom VK, Papoutsi E, Ha DM, et al. Oxidative damage in the gastrocnemius of patients with peripheral artery disease is myofiber type selective. Redox Biol. 2014;2:921-8. https://doi.org/1 0.1016/j.redox.2014.07.002

37. Salmena L, Poliseno L, Tay $Y$, Kats L, Pandolfi PP. A ceRNA hypothesis: the Rosetta stone of a hidden RNA language? Cell. 2011;146(3):353-8. https:// doi.org/10.1016/j.cell.2011.07.014

38. Schuster S, Penke M, Gorski T, Gebhardt R, Weiss TS, Kiess W, et al. FK866induced NAMPT inhibition activates AMPK and downregulates mTOR signaling in hepatocarcinoma cells. Biochem Biophys Res Commun. 2015; 458(2):334-40. https://doi.org/10.1016/j.bbrc.2015.01.111.

39. Tateishi K, lafrate AJ, Ho Q, Curry WT, Batchelor TT, Flaherty KT, et al. Mycdriven glycolysis is a therapeutic target in glioblastoma. Clin Cancer Res. 2016;22(17):4452-65. https://doi.org/10.1158/1078-0432.CCR-15-2274.

40. Nacarelli T, Lau L, Fukumoto T, Zundell J, Fatkhutdinov N, Wu S, et al. $\mathrm{NAD}(+)$ metabolism governs the proinflammatory senescence-associated secretome. Nat Cell Biol. 2019;21(3):397-407. https://doi.org/10.1038/s41556019-0287-4.
41. Kos A, Dijkema R, Arnberg AC, van der Meide PH, Schellekens H. The hepatitis delta (delta) virus possesses a circular RNA. Nature. 1986;323(6088): 558-60. https://doi.org/10.1038/323558a0.

42. Cocquerelle C, Mascrez B, Hetuin D, Bailleul B. Mis-splicing yields circular RNA molecules. FASEB J. 1993;7(1):155-60. https://doi.org/10.1096/fasebj.7.1. 7678559.

43. Danan M, Schwartz S, Edelheit S, Sorek R. Transcriptome-wide discovery of circular RNAs in Archaea. Nucleic Acids Res. 2012;40(7):3131-42. https://doi. org/10.1093/nar/gkr1009.

44. Salzman J, Gawad C, Wang PL, Lacayo N, Brown PO. Circular RNAs are the predominant transcript isoform from hundreds of human genes in diverse cell types. PLoS One. 2012;7(2):e30733. https://doi.org/10.1371/journal.pone. 0030733.

45. Werfel S, Nothjunge S, Schwarzmayr T, Strom TM, Meitinger T, Engelhardt S. Characterization of circular RNAs in human, mouse and rat hearts. J Mol Cell Cardiol. 2016;98:103-7. https://doi.org/10.1016/j.yjmcc.2016.07.007.

46. Zhang L, Liu X, Che S, Cui J, Ma X, An X, et al. Endometrial epithelial cell apoptosis is inhibited by a ciR8073-miR181a-Neurotensis pathway during embryo implantation. Mol Ther Nucleic Acids. 2019;14:262-73. https://doi. org/10.1016/j.omtn.2018.12.005.

47. Jiang R, Li H, Yang J, Shen X, Song C, Yang Z, et al. CircRNA profiling reveals an abundant circFUT10 that promotes adipocyte proliferation and inhibits adipocyte differentiation via sponging let-7. Mol Ther Nucleic Acids. 2020; 20:491-501. https://doi.org/10.1016/j.omtn.2020.03.011.

48. Chen B, Yu J, Guo L, Byers MS, Wang Z, Chen X, et al. Circular RNA circHIPK3 promotes the proliferation and differentiation of chicken myoblast cells by sponging miR-30a-3p. Cells. 2019;8(2):177. https://doi.org/10.3390/cells80201 77.

49. Peng S, Song C, Li H, Cao X, Ma Y, Wang X, et al. Circular RNA SNX29 sponges miR-744 to regulate proliferation and differentiation of myoblasts by activating the Wnt5a/ca (2+) signaling pathway. Mol Ther Nucleic Acids. 2019;16:481-93. https://doi.org/10.1016/j.omtn.2019.03.009.

50. Shen X, Zhang X, Ru W, Huang Y, Lan X, Lei C, et al. CircINSR promotes proliferation and reduces apoptosis of embryonic myoblasts by sponging miR-34a. Mol Ther Nucleic Acids. 2020;19:986-99. https://doi.org/10.1016/j. omtn.2019.12.032

51. Yue B, Yang H, Wu J, Wang J, Ru W, Cheng J, et al. CircSVIL regulates bovine myoblast development by inhibiting STAT1 phosphorylation. Sci China Life Sci. 2021. https://doi.org/10.1007/s11427-020-1908-2.

52. Ju X, Liu Y, Shan Y, Ji G, Zhang M, Tu Y, et al. Analysis of potential regulatory LnCRNAs and CircRNAs in the oxidative myofiber and glycolytic myofiber of chickens. Sci Rep. 2021;11(1):20861. https://doi.org/10.1038/s41 598-021-00176-y.

53. Lin J, Wu H, Tarr PT, Zhang CY, Wu Z, Boss O, et al. Transcriptional coactivator PGC-1 alpha drives the formation of slow-twitch muscle fibres. Nature. 2002;418(6899):797-801. https://doi.org/10.1038/nature00904.

54. Ventura-Clapier R, Garnier A, Veksler V. Transcriptional control of mitochondrial biogenesis: the central role of PGC-1alpha. Cardiovasc Res. 2008;79(2):208-17. https://doi.org/10.1093/cvr/cvn098.

55. Zhang GM, Guo YX, Deng MT, Wan YJ, Deng KP, Xiao SH, et al. Effect of PPARGC1A on the development and metabolism of early rabbit embryos in vitro. Mol Reprod Dev. 2019;86(11):1758-70. https://doi.org/10.1002/ mrd.23269.

Ready to submit your research? Choose BMC and benefit from:

- fast, convenient online submission

- thorough peer review by experienced researchers in your field

- rapid publication on acceptance

- support for research data, including large and complex data types

- gold Open Access which fosters wider collaboration and increased citations

- maximum visibility for your research: over $100 \mathrm{M}$ website views per year

At $\mathrm{BMC}$, research is always in progress.

Learn more biomedcentral.com/submission 